\title{
Intake of fish and marine $n-3$ polyunsaturated fatty acids and risk of breast cancer: meta-analysis of data from 21 independent prospective cohort studies
}

\author{
(c) (1) (8) OPEN ACCESS
}

\author{
Ju-Sheng Zheng PhD student ${ }^{12}$, Xiao-Jie Hu PhD student ${ }^{12}$, Yi-Min Zhao masters student ${ }^{2}$, Jing \\ Yang PhD student ${ }^{12}$, Duo Li professor ${ }^{12}$
}

${ }^{1}$ Department of Food Science and Nutrition, Zhejiang University, Hangzhou 310058, China; ${ }^{2}$ APCNS Center of Nutrition and Food Safety, Hangzhou 310058, China

\begin{abstract}
Objectives To investigate the association between intake of fish and n-3 polyunsaturated fatty acids ( $n-3$ PUFA) and the risk of breast cancer and to evaluate the potential dose-response relation.

Design Meta-analysis and systematic review of prospective cohort studies.

Data sources PubMed and Embase up to December 2012 and references of retrieved relevant articles.

Eligibility criteria for selecting studies Prospective cohort studies with relative risk and $95 \%$ confidence intervals for breast cancer according to fish intake, n-3 PUFA intake, or tissue biomarkers.

Results Twenty six publications, including 20905 cases of breast cancer and 883585 participants from 21 independent prospective cohort studies were eligible. Eleven articles (13 323 breast cancer events and 687770 participants) investigated fish intake, 17 articles investigated marine n-3 PUFA (16 178 breast cancer events and 527392 participants), and 12 articles investigated alpha linolenic acid (14 284 breast cancer events and 405592 participants). Marine n-3 PUFA was associated with 14\% reduction of risk of breast cancer (relative risk for highest $v$ lowest category 0.86 (95\% confidence interval 0.78 to 0.94$\left.), I^{2}=54\right)$, and the relative risk remained similar whether marine $n-3$ PUFA was measured as dietary intake $\left(0.85,0.76\right.$ to $\left.0.96, \mathrm{I}^{2}=67 \%\right)$ or as tissue biomarkers $\left(0.86,0.71\right.$ to $\left.1.03, I^{2}=8 \%\right)$. Subgroup analyses also indicated that the inverse association between marine n-3 PUFA and risk was more evident in studies that did not adjust for body mass index (BMI) $(0.74,0.64$ to $\left.0.86, I^{2}=0\right)$ than in studies that did adjust for $B M I(0.90,0.80$ to 1.01 , $I^{2}=63.2 \%$ ). Dose-response analysis indicated that risk of breast cancer was reduced by $5 \%$ per $0.1 \mathrm{~g} /$ day $\left(0.95,0.90\right.$ to $\left.1.00, \mathrm{I}^{2}=52 \%\right)$ or $0.1 \%$
\end{abstract}

energy/day $\left(0.95,0.90\right.$ to $\left.1.00, I^{2}=79 \%\right)$ increment of dietary marine $n-3$ PUFA intake. No significant association was observed for fish intake or exposure to alpha linolenic acid.

Conclusions Higher consumption of dietary marine n-3 PUFA is associated with a lower risk of breast cancer. The associations of fish and alpha linolenic acid intake with risk warrant further investigation of prospective cohort studies. These findings could have public health implications with regard to prevention of breast cancer through dietary and lifestyle interventions.

\section{Introduction}

Breast cancer is one of the most common cancers and the leading cause of death from cancer among women, accounting for $23 \%$ of the total cancer cases and $14 \%$ of cancer deaths in $2008 .{ }^{1}$ For the past few decades, epidemiological studies ${ }^{23}$ have suggested that a healthy diet and lifestyle is critical for the prevention of breast cancer, and dietary fat is one of the most intensively studied dietary factors closely related with risk. ${ }^{4-8}$ Among subtypes of dietary fat, n-3 polyunsaturated fatty acids (n-3 PUFA) are the most promising types to inhibit or curtail carcinogenesis and reduce risk, as shown in rodent models ${ }^{9}$ and with in vitro cell studies. ${ }^{11}$ Results from observational studies in humans, however, are inconsistent. Several large prospective cohort studies, such as the Singapore Chinese Health Study ${ }^{12}$ and the Japan Collaborative Cohort Study, ${ }^{5}$ have suggested an inverse association between dietary n-3 PUFA intake and risk. Furthermore, several case-control studies have indicated that n-3 PUFA, measured as either dietary intake or with tissue biomarkers, is inversely associated with risk. ${ }^{13-16}$ 
Most of the other cohort or case-control studies, however, found no association between n-3 PUFA and risk. ${ }^{817-27}$ Moreover, accumulating prospective studies suggest that fish, the richest source of marine n-3 PUFA, shows inverse, ${ }^{12} 2829$ null, ${ }^{50213031}$ or even positive $\mathrm{e}^{32} 33$ associations with risk. It is therefore important and interesting to quantitatively assess the association between intake of $n-3$ PUFA and fish and the risk of breast cancer from the available evidence.

We used meta-analysis to summarise the associations between dietary intake of fish and n-3 PUFA with incident breast cancer based on prospective cohort studies. We pooled risk estimates for the highest versus lowest category of intake (or tissue biomarkers) across identified prospective cohort studies to examine the overall association. We conducted a dose-response analysis for the trend estimation and a stratified analysis to examine the sources of heterogeneity.

\section{Methods}

\section{Search strategy and selection criteria}

We followed the criteria for conducting and reporting meta-analysis of observational studies. ${ }^{34}$ A systematic search was conducted in two databases-PubMed and Embase-up to December 2012. We used the following key words treated as title/abstract for the literature search: ("fat" OR "fatty acid" OR "docosahexaenoic acid" OR "eicosapentaenoic acid" OR "docosapentaenoic acid" OR "alpha-linolenic acid" OR "polyunsaturated fatty acid" OR "omega-3 fatty acid" OR "n-3 fatty acid" OR "fish" OR "fish oil" OR "seafood") AND ("breast cancer" OR "breast neoplasms"). Full details of the search strategy are in appendix 1. Our search was restricted to studies in humans and studies published in English. The references of retrieved relevant articles were reviewed to identify potential publications. We did not contact authors for the detailed information of primary studies.

Two investigators (J-SZ and X-JH) independently conducted the literature search, identified potential studies, and extracted detailed information from each included article. Discrepancies were resolved through group discussion with the third investigator (DL). Inclusion criteria were prospective study design (including prospective cohort, nested case-control, and case-cohort studies); the exposure of interest was any type of dietary n-3 PUFA or fish consumption or tissue n-3 PUFA concentrations; the endpoint of interest was incident breast cancer in women; and the risk estimate with corresponding 95\% confidence intervals of breast cancer was reported for n-3 PUFA exposure or fish intake. We excluded retrospective or cross sectional studies, studies in animals, non-original research (reviews, editorials, or commentaries), abstracts, unpublished studies, and duplicated studies.

\section{Data extraction}

From each identified article, we extracted the first author's name, study population and region, study design, duration of follow-up, age of participants, number of cases and non-cases, person years for the population and for each exposure category, risk estimates and corresponding $95 \%$ confidence intervals for each category of n-3 PUFA or fish intake, menopausal status, method of n-3 PUFA measurement (diet or tissue biomarker), and covariates. We extracted risk estimates with the most adjustment.

Quality assessment was conducted according to the Newcastle-Ottawa criteria ${ }^{35}$ for non-randomised studies. A maximum of 9 points was assigned to each study: 4 for selection,
2 for comparability, and 3 for assessment of outcomes (for cohort study) or exposures (for case-control study). We regarded scores of 0-3, 4-6, and 7-9 as low, moderate, and high quality, respectively.

\section{Data synthesis}

We used relative risk for risk estimates, and hazard ratios in cohort studies and odds ratios in nested case-control studies were treated as relative risks directly. We used log transformed relative risk and its corresponding 95\% confidence interval from each eligible study for the meta-analysis. As different studies might use different assessment methods (diet or tissue biomarkers) and report different exposure categories (dichotomous, thirds, quarters, or fifths), we used the study specific relative risk for the highest versus lowest category of fish consumption or n-3 PUFA exposure for the meta-analysis. We then combined the relative risk from each study, weighted by the inverse of their variance, for the meta-analysis with the DerSimonian and Laird random effects model, which takes variation both within and between studies into consideration. ${ }^{36}$ Studies that reported relative risk of breast cancer separately for postmenopausal and premenopausal women ${ }^{12} 193031$ were considered as independent studies for the meta-analysis. Women in one study were reported as either premenopausal or perimenopausal, ${ }^{17}$ and thus we classed them all as premenopausal. One study in which only $10 \%$ of women with breast cancer were premenopausal ${ }^{20}$ was treated as a postmenopausal study. Most of the women with breast cancer in another study were postmenopausal, ${ }^{23}$ and we treated this study as a postmenopausal study.

We conducted meta-analysis for different types of n-3 PUFA separately. Firstly, we estimated the pooled relative risk between the highest versus lowest category of fish intake, total marine n-3 PUFA, alpha linolenic acid (ALA), and total n-3 PUFA, respectively. For studies that did not report a relative risk for total marine n-3 PUFA but that reported risks for eicosapentaenoic acid (EPA), docosahexaenoic acid (DHA), and docosapentaenoic acid (DPA) separately, ${ }^{8}{ }^{17-19} 243738$ we pooled these relative risks to represent the relative risk of total marine n-3 PUFA exposure. One study reported relative risks for intake of dried and non-dried fish separately, ${ }^{29}$ and we pooled the two relative risks with a fixed effects model to get a summary relative risk for total fish intake in this study for further meta-analysis. Secondly, for marine n-3 PUFA, we conducted meta-analysis for EPA, DHA, and EPA separately.

We carried out a dose-response analysis for the trend estimation using generalised least squares regression (two stage GLST in Stata). ${ }^{39}$ For a study without information on the number of cases, number of healthy controls, or person years for exposure categories, we used variance weighted least squares regression for the dose-response estimation. Studies with fewer than three exposure categories were excluded from trend estimation. Dose-response analysis was conducted only in studies that reported dietary intake of n-3 PUFA or fish intake because the results of tissue n-3 PUFA compositions varied according to different tissues (serum, erythrocytes, or adipose) and units used and were not appropriate for standardisation. For fish intake, all the different units were transformed to g/day as described. ${ }^{40}$ One study ${ }^{12}$ reported the unit for the fish intake category as $\mathrm{g} / 1000 \mathrm{kcal}$, which we transformed to g/day assuming an average energy intake of $2000 \mathrm{kcal} /$ day in this population. For dietary n-3 PUFA, we carried out dose-response analysis among studies with exposure units as \% energy/day and g/day, separately. We did not do trend estimation for EPA, DHA, DPA, or total n-3 PUFA because there were limited studies with 
dietary information for these exposures. To estimate a potential curve linear association between fish, dietary n-3 PUFA, and breast cancer, we used a restricted cubic spline model (three knots).

Study heterogeneity was estimated with $\mathrm{I}^{2}$ statistic, with values of $25 \%, 50 \%$, and $75 \%$ representing low, moderate, and high degrees of heterogeneity. Subgroup analysis was conducted to examine sources of study heterogeneity and the influence of potential residual confounding factors, such as age, body mass index (BMI), total energy intake, and education. Univariate meta-regression was performed to examine the significance of the difference in relative risks by different subgroups, including study region (Asian countries $v$ western countries), duration of follow-up (lower $v$ higher than mean duration), exposure measurement (diet $v$ tissue biomarker), study type (cohort $v$ nested case-control), menopausal status (premenopausal $v$ postmenopausal), study quality (score $\leq 7 v>7$ ), risk expression (hazard/rate ratio, relative risk, or odds ratio), and adjustment of covariates (including age, BMI, total energy intake, and education). Sensitivity analysis was conducted by omitting one study at a time and examining the influence of each individual study on the overall relative risk. Publication bias was evaluated by visual inspection of a funnel plot and Egger's regression test (significant at $\mathrm{P}<0.1$ ). We used a trim and fill algorithm ${ }^{41}$ if possible publication bias was detected to identify and correct for the asymmetry of funnel plot from publication bias and provide an adjusted summary relative risk based on all the studies, including the estimated missing studies. Stata version 12 (StataCorp LP, College Station, TX, USA) was used for all the statistical analyses.

\section{Results}

\section{Literature search}

Through full text examination of 215 potential publications we identified 26 eligible publications, including 20905 cases of breast cancer and 883585 participants, from 21 independent prospective cohort studies (fig $1 \Downarrow$ ). Among these articles, 11 were from the United States, ${ }^{6} 819-2124303238424311$ from Europe, ${ }^{1718232526283133374445}$ and four from Asia. ${ }^{512} 2729$ Eleven articles described the association between fish intake and risk of breast cancer, ${ }^{512} 202128-334217$ described the association between marine n-3 PUFA intake and risk, ${ }^{5} 812$ 17-21 23-27 37384344 12 reported the association between ALA and risk, ${ }^{8} 12$ 17-19 23-25 27373844 and 10 described the association between total n-3 PUFA and risk. ${ }^{5812181923-254445}$

\section{Study characteristics}

The 26 eligible articles were prospective cohort studies with mean follow-up ranging from 4.3 to 20 years. Among these, eight were nested case-control studies ${ }^{17-19} 2324424445$ and two were case-cohort studies ${ }^{2637}$ (see table A in appendix 2 and table $\mathrm{B}$ in appendix 3). For two cohorts, relative risks for fish intake and n-3 PUFA were reported in two different publications. ${ }^{19263342}$ For one cohort, the relative risks for fish intake, n-3 PUFA intake, and tissue n-3 PUFA concentrations were reported in three different articles. ${ }^{23}{ }^{25} 31$ One study reported the relative risk for additional breast cancer events in a cohort of women who had received a diagnosis and been treated for early stage breast cancer. ${ }^{43}$ One study reported only the relative risk for the percentage change of dietary n-3 PUFA, ${ }^{6}$ and thus it was included only in the dose-response analysis. The average score for the quality assessment of included studies was 7.6, and the score for all the studies was 5 or above (moderate or high quality) (see supplemental table $\mathrm{C}$ in appendix 3 ).

\section{Fish consumption and risk of breast cancer}

Eleven studies from 11 independent cohorts reported an association between fish intake and risk of breast cancer, with 13323 breast cancer events and 687770 participants. Overall, fish intake was not associated with risk (relative risk 1.03, 95\% confidence interval 0.93 to 1.14 ) (fig $2 \Downarrow$ ). There was moderate study heterogeneity $\left(I^{2}=54 \%\right)$. No publication bias was observed from the funnel plot (see supplemental fig $\mathrm{A}$ in appendix 4 ) or Eggers test $(\mathrm{P}=0.6)$.

All eleven studies were eligible for the trend estimation. Dose-response analysis found no association with risk of breast cancer per $15 \mathrm{~g} /$ day increment of fish intake (relative risk 1.00, $95 \%$ confidence interval 0.97 to 1.03 ) (see supplemental fig B in appendix 4). No publication bias was observed. We did not find a curvilinear association between fish intake and risk ( $\mathrm{P}=0.22$ for non-linearity) (see supplemental fig $\mathrm{C}$ in appendix 4).

\section{Marine n-3 PUFA and risk of breast cancer}

Seventeen articles from 16 independent cohort studies reported the association between marine n-3 PUFA and risk of breast cancer, involving 16178 breast cancer events and 527392 participants. Marine n-3 PUFA was inversely associated with risk (relative risk $0.86,95 \%$ confidence interval 0.78 to 0.94 ; $\mathrm{I}^{2}=54 \%$ ) (fig $3 \Downarrow$ ). The funnel plot (see supplemental fig D in appendix 4) and Eggers test $(\mathrm{P}=0.017)$ indicated slight publication bias. Trim and fill analysis, however, did not change the result.

Eight articles were eligible for the dose-response analysis of dietary marine n-3 PUFA and risk of breast cancer. ${ }^{5612} 2021252743$ Three studies reported marine n-3 PUFA as g/day, ${ }^{21}{ }^{27}{ }^{43}$ while the five other studies ${ }^{5122025}$ reported marine n-3 PUFA as \% energy intake/day. Dose-response analysis indicated that a $0.1 \mathrm{~g} /$ day increment of dietary marine n-3 PUFA was associated with $5 \%$ lower risk of breast cancer (relative risk $0.95,95 \%$ confidence interval 0.90 to $1.00, \mathrm{I}^{2}=52 \%$ ). A $0.1 \%$ energy increment of daily dietary marine n-3 PUFA was inversely associated $5 \%$ reduction of risk $\left(0.95,0.90\right.$ to $\left.1.00, \mathrm{I}^{2}=79 \%\right)$ (fig $4 \Downarrow$ ). There was no significant curvilinear association between marine $\mathrm{n}-3$ PUFA ( $\mathrm{g} /$ day) and risk $(\mathrm{P}=0.21$ for non-linearity, fig $5 \Downarrow$ ). For studies with n-3 PUFA measured as $\%$ energy/day, however, we observed a significant curvilinear association ( $\mathrm{P}=0.011$ for non-linearity, fig $6 \Downarrow$ ).

We then summarised the relative risks for EPA, DHA, and DPA from identified studies. Ten articles ${ }^{8} 17-19232426373844$ reported relative risk for both EPA and DHA, while four articles ${ }^{19} 242644$ reported relative risk for DPA. There were marginally significant inverse associations for EPA (relative risk $0.93,95 \%$ confidence interval 0.85 to 1.02$)$ and DHA $(0.88,0.75$ to 1.03$)$ and risk. No significant association was found for DPA and risk (see supplemental table D in appendix 3).

\section{ALA, total n-3 PUFA, and risk of breast cancer}

Twelve articles, involving 14284 breast cancer events and 405 592 participants, were included in the analysis of association between ALA exposure and risk of breast cancer. We found no significant association between ALA and risk (relative risk 0.97, 95\% confidence interval 0.90 to 1.04) (see supplemental fig E in appendix 4) and no study heterogeneity $\left(\mathrm{I}^{2}=0\right)$ or publication bias ( $\mathrm{P}=0.37$ for Egger's test) (see supplemental fig $\mathrm{F}$ in appendix 4). Dose-response analysis showed no significant association with breast cancer per $0.1 \mathrm{~g} /$ day increment of dietary ALA intake (0.99, 0.98 to 1.01$)$ or per $0.1 \%$ energy/day increment of dietary ALA intake (1.00, 0.99 to 1.00$)$ (see 
supplemental fig $\mathrm{G}$ in appendix 4). No curvilinear association was observed for dietary ALA intake and risk (data not shown).

Ten articles reported relative risk for total n-3 PUFA and risk of breast cancer, and there was no significant association $(0.96$, 0.86 to $\left.1.06, \mathrm{I}^{2}=13 \%\right)$. Slight publication bias was observed ( $\mathrm{P}=0.04$ for Eggers test; see supplemental fig $\mathrm{H}$ in appendix 4); however the results remained unchanged after trim and fill analysis.

\section{Subgroup analysis}

For fish intake and ALA exposure, meta-regression and subgroup analyses did not show any substantial change in the summary relative risk (table $\Downarrow$, and supplemental table $\mathrm{E}$ in appendix 3). Exclusion of any individual study did not change the results. Total n-3 PUFA, however, was significantly inversely associated with risk (relative risk $0.77,95 \%$ confidence interval 0.60 to 0.99 ) only in studies without adjustment for BMI, and no association was observed for studies with adjustment for BMI.

For total marine n-3 PUFA, the inverse association was present in both Asian countries and western countries, though it was more evident in Asian countries (relative risk 0.69, 95\% confidence interval 0.56 to 0.85 ) and no study heterogeneity was observed $\left(I^{2}=0\right)$ (table $\left.\downarrow\right)$. Stratified by different measurement methods of n-3 PUFA, the relative risk for dietary n-3 PUFA was similar to that of tissue biomarkers, while study heterogeneity was much lower in studies measured as biomarkers $\left(\mathrm{I}^{2}=8 \%\right.$ for diet $v 67 \%$ for biomarker). The inverse association between marine n-3 PUFA and risk was more evident in studies without adjustment for BMI $(0.74,0.64$ to $0.86)$ compared with studies with such adjustment $(0.90,0.80$ to 1.01$)$.

For individual marine n-3 PUFA, the significant inverse association with risk of breast cancer was observed only in studies with shorter follow-up (relative risk $0.82(95 \%$ confidence interval 0.70 to 0.96 ) for EPA and 0.74 (0.62 to 0.89 ) for DHA; see supplemental table D in appendix 3), while it was not significant for either n-3 PUFA among studies with longer follow-up, which could be attributed to the limited number of studies with long follow-up. In addition, for both EPA and DHA exposure, their inverse associations with risk were more evident in studies that adjusted for BMI or education compared with studies without such adjustment.

\section{Discussion}

In this meta-analysis dietary intake of marine n-3 polyunsaturated fatty acids (PUFA), but not alpha linolenic acid (ALA), was associated with a lower risk of breast cancer. Fish consumption was not associated with risk. Dose-response analyses indicated a $5 \%$ lower risk of breast cancer per $0.1 \mathrm{~g} /$ day or $0.1 \%$ energy/day increment of dietary marine n-3 PUFA, but no significant trend for ALA or fish intake. To the best of our knowledge, this is the first time meta-analysis has systematically and quantitatively evaluated the association between intake of fish and n-3 PUFA and risk of breast cancer.

\section{Results in relation to other studies}

Ecological studies ${ }^{46} 47$ and prospective cohort studies ${ }^{12} 2829$ have suggested an inverse association between fish consumption and risk of breast cancer and mortality. Marine n-3 PUFA (EPA, DHA, and DPA) are abundant in fish fat, and a few large prospective cohort studies ${ }^{52}$ and case-control studies ${ }^{13-16}$ have reported an inverse association with risk of breast cancer. These findings all agreed with one meta-analysis ${ }^{4}$ based on studies that assessed the biomarkers of intakes of dietary fatty acids, which suggested a potential protective effect of marine n-3 PUFA on breast cancer. Another systematic review suggested that there was no protective association between n-3 PUFA and breast cancer. ${ }^{7}$ Further evidence has been published since then, and quite a few of the newly published prospective studies indicated potential protective effects of n-3 PUFA on breast cancer. ${ }^{27} 3843$ Our meta-analysis based on this evidence, together with previous publications, supports a protective role of marine n-3 PUFA on the incidence of breast cancer.

Subgroup analysis indicated that the protective effect of marine n-3 PUFA was more evident in Asian countries than in the US or European countries. Fish intake also tended to be associated with a lower risk of breast cancer in Asian populations, rather than in western populations. This could be because typical fish intake is much higher in Asian populations than in western populations. ${ }^{48}$ Therefore fish intake in these western populations might be too low to detect an expected protective effect.

Furthermore, in North America and some European countries, a large proportion of intake of marine n-3 PUFA probably comes from fish oil supplementation in the form of capsules, thereby contributing to the different effects on risk. In addition, the protective effect of fish intake might be attenuated or even reversed by other constituents in fish, such as organometallics and pesticides. Taken together, these factors could explain our finding of an overall null association between fish intake and risk of breast cancer, which was in line with a previous large pooled analysis. ${ }^{49}$

Tissue n-3 PUFA concentrations, compared with dietary assessment, might provide a more accurate estimation of intake. Subgroup analysis for marine n-3 PUFA and risk, however, indicated that the summary risk estimate for studies with dietary information was similar to that of tissue biomarkers, which further confirmed the robust results of the present meta-analysis. Further concerns regarding n-3 PUFA and risk are menopausal status and hormone receptor status (oestrogen receptor and progesterone receptor). Previous cohort studies indicated that the protective effect of marine n-3 PUFA against breast cancer was more evident in postmenopausal women than in premenopausal women. ${ }^{12}{ }^{19}$ Our meta-analysis confirmed that n-3 PUFA intake was significantly inversely associated with breast cancer in postmenopausal but not in premenopausal women; this could mean that any benefit of marine n-3 PUFA is usually after long term exposure, which could be observed best at the postmenopausal period because breast cancer is a disease with a long latency between exposure and development. Another explanation could be related to the different effects of body fat on premenopausal and postmenopausal risk of breast cancer. One recent meta-analysis suggested that high BMI tends to be protective against premenopausal breast cancer but is a risk factor for postmenopausal breast cancer, ${ }^{50}$ and an interaction between BMI and menopausal status on breast cancer has been proposed. Therefore, marine n-3 PUFA could influence risk through BMI, which was supported by our subgroup analysis. The inverse association between marine n-3 PUFA and risk was greatly attenuated in studies that adjusted for BMI compared with studies without such adjustment. Most of the studies that investigated marine n-3 PUFA intake and postmenopausal breast cancer, however, did adjust for BMI, and an overall significant inverse association between marine n-3 PUFA and risk still existed in these postmenopausal studies. This suggests that the effect of marine n-3 PUFA on risk was partly independent of BMI, and a more precise mechanism for this discrepancy remains to be investigated. In addition, only a few studies 
examined the influence of oestrogen receptor and progesterone receptor status on the association between fish intake and risk of breast cancer. Stripp and colleagues reported an adverse effect of fish consumption only for oestrogen receptor positive breast cancer in a cohort of postmenopausal women. ${ }^{33}$ Two other studies based on large prospective cohorts found no evidence for the influence of hormone receptor status on n-3 PUFA and risk. ${ }^{6}$ More prospective studies are warranted to investigate the impact of hormone receptor status.

In contrast with marine n-3 PUFA, the effect of ALA, a plant based n-3 PUFA, on breast tumour growth is less clear, and we found no significant association. The explanation for the inconsistency among studies regarding dietary ALA and risk of breast cancer could be the different dietary sources. Thiebaut and colleagues showed that dietary ALA from fruit and vegetables and vegetable oils was inversely associated with risk but observed a positive association for ALA from nut mixes and processed meat. ${ }^{25}$ In addition, the biological effect of ALA on breast cancer per se might be not as strong as marine n-3 PUFA, as suggested by the summarised relative risk of biomarker data with no significant association in our study. Taken together, we found no significant protective association with ALA, and the inconsistent associations observed among previous studies might reflect different dietary/food patterns involving other nutrients related to risk of breast cancer.

The anticarcinogenic effects of marine n-3 PUFA are biologically plausible. Possible mechanisms include inhibition of eicosanoid derived from arachidonic acid, regulation of transcription factor activity, gene expression and activities of molecules involved in the signal transduction of cell growth, differentiation apoptosis, angiogenesis, and metastasis. ${ }^{51}$ In addition, marine n-3 PUFA could decrease the production of oestrogen, thus reducing oestrogen stimulated cell growth. ${ }^{51}$ Specifically, studies using cell lines and on rodent models have shown the protective effects of marine n-3 PUFA against breast tumour growth. ${ }^{9-1152}$

\section{Strengths and limitations}

The present meta-analysis has several strengths. Firstly, the large sample size allowed us to quantitatively assess the association of fish and n-3 PUFA intake and risk of breast cancer, thus making it more powerful than any individual study. Secondly, the prospective nature of the included studies avoided the influence of recall and selection bias. Thirdly, we systematically reviewed and assessed the summarised association between breast cancer with different types of individual n-3 PUFA, including EPA, DHA, DPA, and ALA. These data gave a most comprehensive view of the association between n-3 fatty acids and risk based on the current evidence.

The meta-analysis does, however, also have several limitations. Firstly, different methods of assessment (diet and tissue biomarker) were used in the included studies, and the units were heterogeneous across different studies. Nevertheless, we used relative risks for the highest versus lowest category of $n-3$ or fish intake, which could, to some extent, reduce the bias caused by different units or exposure assessment methods. Furthermore, dose-response analysis supports our results. Secondly, available data on the individual n-3 PUFA, especially DPA, is rather limited. Therefore, future prospective studies are needed for the detailed analysis of association between individual n-3 PUFA and risk of breast cancer. Thirdly, the observational nature of the included studies makes it subject to the influence of residual confounders. In addition, possible language bias could occur because we excluded articles not in English. Our eligible articles, however, covered a wide range of non-English countries, such as countries across Europe and Asia, and the number of large cohorts in other non-English countries is limited.

\section{Conclusions}

Our findings have important public health implications. The prevention of breast cancer continues to be an important public health issue for researchers, especially with regard to the investigation of relations between breast cancer, diet, and lifestyle. Evidence from either experimental or observational studies suggests a protective effect of marine n-3 PUFA on breast cancer, though no conclusive results have been achieved. Systematic review and meta-analysis are the most powerful tools to assess these kinds of inconsistent associations. Therefore, our present study provides solid and robust evidence that marine n-3 PUFA are inversely associated with risk of breast cancer. The protective effect of fish or individual n-3 PUFA warrants further investigation of prospective studies.

We thank Laurence D Parnell at Jean Mayer USDA Human Nutrition Research Center on Aging at Tufts University for helping to edit the manuscript.

Contributors: J-SZ and DL conceived the study. J-SZ and X-JH searched the databases and checked them according to the eligible criteria and exclusion criteria. DL helped develop search strategies. J-SZ analysed the data and wrote the draft of the paper. All authors contributed to writing, reviewing, or revising the paper. DL is guarantor.

Funding: This study was funded by National Natural Science Foundation of China (NSFC, No 81273054), the PhD. Programs Foundation of Ministry of Education of China (20120101110107), and the National Basic Research Program of China (973 Program 2011CB504002). The funder had no role in study design, data collection and analysis, decision to publish, or preparation of the manuscript.

Competing interests: All authors have completed the ICMJE uniform disclosure form at www.icmje.org/coi_disclosure.pdf (available on request from the corresponding author) and declare: no support from any organisation for the submitted work; no financial relationships with any organisations that might have an interest in the submitted work in the previous three years; no other relationships or activities that could appear to have influenced the submitted work.

Ethical approval: Not required.

Data sharing: No additional data available.

1 Jemal A, Bray F, Center MM, Ferlay J, Ward E, Forman D. Global cancer statistics. $C A$ Cancer J Clin 2011;61:69-90.

2 Brennan SF, Cantwell MM, Cardwell CR, Velentzis LS, Woodside JV. Dietary patterns and breast cancer risk: a systematic review and meta-analysis. Am J Clin Nutr 2010;91:1294-302.

3 Lambrechts S, Decloedt J, Neven P. Breast cancer prevention: lifestyle changes and chemoprevention. Acta Clin Belg 2011;66:283-92.

4 Saadatian-Elahi M, Norat T, Goudable J, Riboli E. Biomarkers of dietary fatty acid intake and the risk of breast cancer: a meta-analysis. Int J Cancer 2004;111:584-91.

5 Wakai K, Tamakoshi K, Date C, Fukui M, Suzuki S, Lin Y, et al. Dietary intakes of fat and fatty acids and risk of breast cancer: a prospective study in Japan. Cancer $\mathrm{Sci}$ 2005;96:590-9.

6 Kim EH, Willett WC, Colditz GA, Hankinson SE, Stampfer MJ, Hunter DJ, et al. Dietary fat and risk of postmenopausal breast cancer in a 20-year follow-up. Am J Epidemiol 2006;164:990-7.

7 MacLean CH, Newberry SJ, Mojica WA, Khanna P, Issa AM, Suttorp MJ, et al. Effects of omega-3 fatty acids on cancer risk: a systematic review. JAMA 2006:295:403-15.

8 Park SY, Kolonel LN, Henderson BE, Wilkens LR. Dietary fat and breast cancer in postmenopausal women according to ethnicity and hormone receptor status: the Multiethnic Cohort Study. Cancer Prev Res (Phila) 2012;5:216-28.

9 Karmali RA, Marsh J, Fuchs C. Effect of omega-3 fatty acids on growth of a rat mammary tumor. J Natl Cancer Inst 1984;73:457-61.

10 Kort WJ, Weijma IM, Bijma AM, van Schalkwijk WP, Vergroesen AJ, Westbroek DL. Omega-3 fatty acids inhibiting the growth of a transplantable rat mammary adenocarcinoma. J Natl Cancer Inst 1987;79:593-9.

11 Schley PD, Jijon HB, Robinson LE, Field CJ. Mechanisms of omega-3 fatty acid-induced growth inhibition in MDA-MB-231 human breast cancer cells. Breast Cancer Res Treat 2005;92:187-95.

12 Gago-Dominguez M, Yuan JM, Sun CL, Lee HP, Yu MC. Opposing effects of dietary n-3 and n-6 fatty acids on mammary carcinogenesis: the Singapore Chinese Health Study. Br J Cancer 2003;89:1686-92. 


\section{What is already known on this topic}

Breast cancer is one of the most common cancers and the leading cause of cancer death among women in the world

Epidemiological studies suggest that fish and n-3 polyunsaturated fatty acids intake have a protective role in breast cancer, though

results from prospective studies are inconsistent

\section{What this study adds}

High intake of marine n-3 polyunsaturated fatty acids is associated with $14 \%$ reduction in risk of breast cancer

Each $0.1 \mathrm{~g} /$ day or $0.1 \%$ energy/day increment of intake was associated with a $5 \%$ reduction in risk

13 Kim J, Lim SY, Shin A, Sung MK, Ro J, Kang HS, et al. Fatty fish and fish omega-3 fatty acid intakes decrease the breast cancer risk: a case-control study. BMC Cancer 2009;9:216.

14 Shannon J, King IB, Moshofsky R, Lampe JW, Gao DL, Ray RM, et al. Erythrocyte fatty acids and breast cancer risk: a case-control study in Shanghai, China. Am J Clin Nutr 2007;85:1090-7.

15 Kuriki K, Hirose K, Wakai K, Matsuo K, Ito H, Suzuki T, et al. Breast cancer risk and erythrocyte compositions of n-3 highly unsaturated fatty acids in Japanese. Int $\mathrm{J}$ Cancer 2007;121:377-85.

16 Maillard V, Bougnoux P, Ferrari P, Jourdan ML, Pinault M, Lavillonniere F, et al. N-3 and $\mathrm{N}-6$ fatty acids in breast adipose tissue and relative risk of breast cancer in a case-control study in Tours, France. Int $J$ Cancer 2002;98:78-83.

17 Vatten LJ, Bjerve KS, Andersen A, Jellum E. Polyunsaturated fatty acids in serum phospholipids and risk of breast cancer: a case-control study from the Janus serum bank in Norway. Eur J Cancer 1993;29A:532-8.

18 Chajes V, Hulten K, Van Kappel AL, Winkvist A, Kaaks R, Hallmans G, et al. Fatty-acid composition in serum phospholipids and risk of breast cancer: an incident case-control study in Sweden. Int J Cancer 1999;83:585-90.

19 Saadatian-Elahi M, Toniolo P, Ferrari P, Goudable J, Akhmedkhanov A Zeleniuch-Jacquotte $A$, et al. Serum fatty acids and risk of breast cancer in a nested case-control study of the New York University Women"s Health Study. Cancer Epidemiol Biomarkers Prev 2002;11:1353-60.

20 Cho E, Spiegelman D, Hunter DJ, Chen WY, Stampfer MJ, Colditz GA, et al. Premenopausal fat intake and risk of breast cancer. J Natl Cancer Inst 2003:95:1079-85.

21 Folsom AR, Demissie Z. Fish intake, marine omega-3 fatty acids, and mortality in a cohort of postmenopausal women. Am J Epidemiol 2004;160:1005-10.

22 Wirfalt E, Vessby B, Mattisson I, Gullberg B, Olsson H, Berglund G. No relations between breast cancer risk and fatty acids of erythrocyte membranes in postmenopausal women of the Malmo Diet Cancer cohort (Sweden). Eur J Clin Nutr 2004;58:761-70.

23 Chajes V, Thiebaut AC, Rotival M, Gauthier E, Maillard V, Boutron-Ruault MC, et al. Association between serum trans-monounsaturated fatty acids and breast cancer risk in the E3N-EPIC Study. Am J Epidemiol 2008;167:1312-20.

24 Takata Y, King IB, Neuhouser ML, Schaffer S, Barnett M, Thornquist M, et al. Association of serum phospholipid fatty acids with breast cancer risk among postmenopausal cigarette smokers. Cancer Causes Control 2009;20:497-504.

25 Thiebaut AC, Chajes V, Gerber M, Boutron-Ruault MC, Joulin V, Lenoir G, et al. Dietary intakes of omega- 6 and omega- 3 polyunsaturated fatty acids and the risk of breast cancer. Int J Cancer 2009;124:924-31.

26 Witt PM, Christensen JH, Schmidt EB, Dethlefsen C, Tjonneland A, Overvad K, et al. Marine $n-3$ polyunsaturated fatty acids in adipose tissue and breast cancer risk: a case-cohort study from Denmark. Cancer Causes Control 2009;20:1715-21.

27 Murff HJ, Shu XO, Li H, Yang G, Wu X, Cai H, et al. Dietary polyunsaturated fatty acids and breast cancer risk in Chinese women: a prospective cohort study. Int $\mathrm{J}$ Cancer 2011;128:1434-41.

28 Vatten LJ, Solvoll K, Loken EB. Frequency of meat and fish intake and risk of breast cancer in a prospective study of 14,500 Norwegian women. Int J Cancer 1990;46:12-5.

29 Key TJ, Sharp GB, Appleby PN, Beral V, Goodman MT, Soda M, et al. Soya foods and breast cancer risk: a prospective study in Hiroshima and Nagasaki, Japan. Br J Cancer 1999;81:1248-56.

30 Holmes MD, Colditz GA, Hunter DJ, Hankinson SE, Rosner B, Speizer FE, et al. Meat, fish and egg intake and risk of breast cancer. Int J Cancer 2003;104:221-7.

31 Engeset D, Alsaker E, Lund E, Welch A, Khaw KT, Clavel-Chapelon F, et al. Fish consumption and breast cancer risk. The European Prospective Investigation into Cancer and Nutrition (EPIC). Int $J$ Cancer 2006;119:175-82.

32 Mills PK, Beeson WL, Phillips RL, Fraser GE. Dietary habits and breast cancer incidence among Seventh-day Adventists. Cancer 1989;64:582-90.

33 Stripp C, Overvad K, Christensen J, Thomsen BL, Olsen A, Moller S, et al. Fish intake is positively associated with breast cancer incidence rate. J Nutr 2003;133:3664-9.
34 Stroup DF, Berlin JA, Morton SC, Olkin I, Williamson GD, Rennie D, et al. Meta-analysis of observational studies in epidemiology: a proposal for reporting. Meta-analysis of observational studies in epidemiology (MOOSE) group. JAMA 2000;283:2008-12.

35 Wells GA, Shea B, O"Connell D, Peterson J, Welch V, Losos M, et al. The Newcastle-Ottawa Scale (NOS) for assessing the quality of nonrandomised studies in meta-analyses. 2011. www.ohri.ca/programs/clinical epidemiology/oxford.htm.

36 DerSimonian R, Laird N. Meta-analysis in clinical trials. Control Clin Trials 1986;7:177-88.

37 Voorrips LE, Brants HA, Kardinaal AF, Hiddink GJ, van den Brandt PA, Goldbohm RA. Intake of conjugated linoleic acid, fat, and other fatty acids in relation to postmenopausal breast cancer: the Netherlands Cohort Study on Diet and Cancer. Am J Clin Nutr 2002;76:873-82.

38 Sczaniecka AK, Brasky TM, Lampe JW, Patterson RE, White E. Dietary intake of specific fatty acids and breast cancer risk among postmenopausal women in the VITAL Cohort. Nutr Cancer 2012;64:1131-42

39 Orsini N, Bellocco R, Greenland S. Generalized least squares for trend estimation of summarized dose-response data. Stata J 2006;6:40-57.

40 Zheng J, Huang T, Yu Y, Hu X, Yang B, Li D. Fish consumption and CHD mortality: an updated meta-analysis of seventeen cohort studies. Public Health Nutr 2011;15:725-37.

41 Duval S, Tweedie R. Trim and fill: a simple funnel-plot-based method of testing and adjusting for publication bias in meta-analysis. Biometrics 2000;56:455-63.

42 Toniolo P, Riboli E, Shore RE, Pasternack BS. Consumption of meat, animal products, protein, and fat and risk of breast cancer: a prospective cohort study in New York. Epidemiology 1994:5:391-7.

43 Patterson RE, Flatt SW, Newman VA, Natarajan L, Rock CL, Thomson CA, et al. Marine fatty acid intake is associated with breast cancer prognosis. $J$ Nutr 2011;141:201-6.

44 Pala V, Krogh V, Muti P, Chajes V, Riboli E, Micheli A, et al. Erythrocyte membrane fatty acids and subsequent breast cancer: a prospective Italian study. J Nat/ Cancer Inst 2001;93:1088-95.

45 Wirfalt E, Mattisson I, Gullberg B, Johansson U, Olsson H, Berglund G. Postmenopausal breast cancer is associated with high intakes of omega6 fatty acids (Sweden). Cancer Causes Control 2002;13:883-93.

46 Kaizer L, Boyd NF, Kriukov V, Tritchler D. Fish consumption and breast cancer risk: an ecological study. Nutr Cancer 1989;12:61-8.

47 Sasaki S, Horacsek M, Kesteloot H. An ecological study of the relationship between dietary fat intake and breast cancer mortality. Prev Med 1993;22:187-202.

48 Terry PD, Rohan TE, Wolk A. Intakes of fish and marine fatty acids and the risks of cancers of the breast and prostate and of other hormone-related cancers: a review of the epidemiologic evidence. Am J Clin Nutr 2003;77:532-43.

49 Missmer SA, Smith-Warner SA, Spiegelman D, Yaun SS, Adami HO, Beeson WL, et al. Meat and dairy food consumption and breast cancer: a pooled analysis of cohort studies. Int J Epidemiol 2002;31:78-85.

50 Cheraghi Z, Poorolajal J, Hashem T, Esmailnasab N, Doosti Irani A. Effect of body mass index on breast cancer during premenopausal and postmenopausal periods: a meta-analysis. PLoS One 2012;7:e51446.

51 Larsson SC, Kumlin M, Ingelman-Sundberg M, Wolk A. Dietary long-chain n-3 fatty acids for the prevention of cancer: a review of potential mechanisms. Am J Clin Nutr 2004;79:935-45.

52 Cave WT, Jr. Omega-3 polyunsaturated fatty acids in rodent models of breast cancer. Breast Cancer Res Treat 1997;46:239-46.

\section{Accepted: 31 May 2013}

\section{Cite this as: BMJ 2013;346:f3706}

This is an Open Access article distributed in accordance with the Creative Commons Attribution Non Commercial (CC BY-NC 3.0) license, which permits others to distribute, remix, adapt, build upon this work non-commercially, and license their derivative works on different terms, provided the original work is properly cited and the use is non-commercial. See: http://creativecommons.org/licenses/by-nc/3.0/. 


\section{Table}

Table 1/ Subgroup analyses of intake of fish and marine n-3 polyunsaturated fatty acids (PUFA) and risk of breast cancer (highest versus lowest category)

\begin{tabular}{|c|c|c|c|c|c|c|}
\hline \multirow[b]{2}{*}{ Subgroup } & \multicolumn{3}{|c|}{ Fish intake } & \multicolumn{3}{|c|}{ Marine n-3 PUFA } \\
\hline & No of studies & Relative risk (95\% Cl) & $I^{2}(\%)$ & No of studies & Relative risk (95\% Cl) & $I^{2}(\%)$ \\
\hline Overall & 14 & 1.03 (0.93 to 1.14$)$ & 54 & 19 & 0.86 (0.78 to 0.94$)$ & 54 \\
\hline \multicolumn{7}{|l|}{ Regions: } \\
\hline Asian countries & 4 & 0.84 (0.65 to 1.08$)$ & 40 & 4 & $0.69(0.56$ to 0.85$)$ & 0 \\
\hline Western countries & 10 & $1.08(0.96$ to 1.20$)$ & 54 & 15 & $0.89(0.81$ to 0.98$)$ & 52 \\
\hline US & 6 & $1.05(0.92$ to 1.20$)$ & 53 & 8 & $0.87(0.76$ to 1.00$)$ & 67 \\
\hline European countries & 4 & $1.11(0.90$ to 1.37$)$ & 59 & 7 & $0.93(0.80$ to 1.07$)$ & 23 \\
\hline \multicolumn{7}{|l|}{ Duration of follow-up (years)* } \\
\hline$\leq$ Mean & 9 & 1.05 (0.88 to 1.24$)$ & 63 & 11 & 0.79 (0.71 to 0.88$)$ & 0 \\
\hline$>$ Mean & 5 & $1.00(0.90$ to 1.10$)$ & 16 & 8 & 0.91 (0.81 to 1.03 ) & 61 \\
\hline \multicolumn{7}{|l|}{ Menopausal status: } \\
\hline Premenopausal & 4 & $1.04(0.91$ to 1.20$)$ & 0 & 4 & 0.96 (0.78 to 1.18$)$ & 0 \\
\hline Postmenopausal & 6 & $1.08(0.92$ to 1.27$)$ & 73 & 10 & $0.88(0.76$ to 1.00$)$ & 65 \\
\hline Combined & 4 & 0.87 (0.67 to 1.12$)$ & 38 & 5 & $0.77(0.64$ to 0.93$)$ & 47 \\
\hline \multicolumn{7}{|l|}{ Study type: } \\
\hline Prospective cohort & 13 & $1.03(0.92$ to 1.14$)$ & 57 & 10 & $0.84(0.74$ to 0.95$)$ & 70 \\
\hline Nested case-control & 1 & $1.02(0.61$ to 1.71$)$ & - & 7 & $0.83(0.67$ to 1.03$)$ & 8 \\
\hline Case-cohort & 0 & - & - & 2 & $0.98(0.81$ to 1.20$)$ & 0 \\
\hline \multicolumn{7}{|l|}{ Measurement method: } \\
\hline Dietary intake & 14 & $1.03(0.93$ to 1.14$)$ & 54 & 11 & 0.85 (0.76 to 0.96$)$ & 67 \\
\hline Biomarker & 0 & - & - & 8 & 0.86 (0.71 to 1.03$)$ & 8 \\
\hline \multicolumn{7}{|l|}{ Covariate adjustment: } \\
\hline Adjustment for age & 10 & $0.97(0.85$ to 1.10$)$ & 56 & 11 & $0.86(0.77$ to 0.97$)$ & 64 \\
\hline No adjustment for age & 4 & 1.15 (1.02 to 1.31$)$ & 9 & 8 & 0.83 (0.70 to 0.98$)$ & 16 \\
\hline Adjustment for BMI & 7 & $1.07(0.91$ to 1.26$)$ & 70 & 11 & 0.90 (0.80 to 1.01$)$ & 63 \\
\hline No adjustment for BMI & 7 & $0.99(0.87$ to 1.13$)$ & 25 & 8 & $0.74(0.64$ to 0.86$)$ & 0 \\
\hline Adjustment for energy & 6 & 0.98 (0.89 to 1.08$)$ & 15 & 7 & 0.87 (0.74 to 1.01$)$ & 5 \\
\hline No adjustment for energy & 8 & $1.08(0.91$ to 1.28$)$ & 62 & 12 & 0.85 (0.77 to 0.94$)$ & 76 \\
\hline Adjustment for education & 6 & $1.00(0.75$ to 1.34$)$ & 77 & 10 & $0.86(0.75$ to 1.00$)$ & 69 \\
\hline No adjustment for education & 8 & $1.03(0.96$ to 1.11$)$ & 0 & 9 & 0.87 (0.78 to 0.96$)$ & 0 \\
\hline \multicolumn{7}{|l|}{ Study quality: } \\
\hline Score $>7$ & 8 & $0.98(0.85$ to 1.14$)$ & 61 & 11 & 0.86 (0.77 to 0.97$)$ & 64 \\
\hline Score $\leq 7$ & 6 & $1.10(0.95$ to 1.26$)$ & 35 & 8 & 0.83 (0.70 to 0.98$)$ & 16 \\
\hline \multicolumn{7}{|l|}{ Risk expression: } \\
\hline Hazard/rate ratio & 5 & 1.06 (0.89 to 1.27$)$ & 59 & 7 & 0.90 (0.79 to 1.03$)$ & 70 \\
\hline Relative risk & 8 & $1.00(0.86$ to 1.16$)$ & 60 & 5 & $0.77(0.63$ to 0.94$)$ & 37 \\
\hline Odds ratio & 1 & $1.02(0.61$ to 1.71$)$ & - & 7 & 0.83 (0.67 to 1.03$)$ & 17 \\
\hline
\end{tabular}

${ }^{*}$ Mean duration of follow-up was 9.4 years for fish intake, and 6.8 years for marine $n-3$ PUFA. 


\section{Figures}

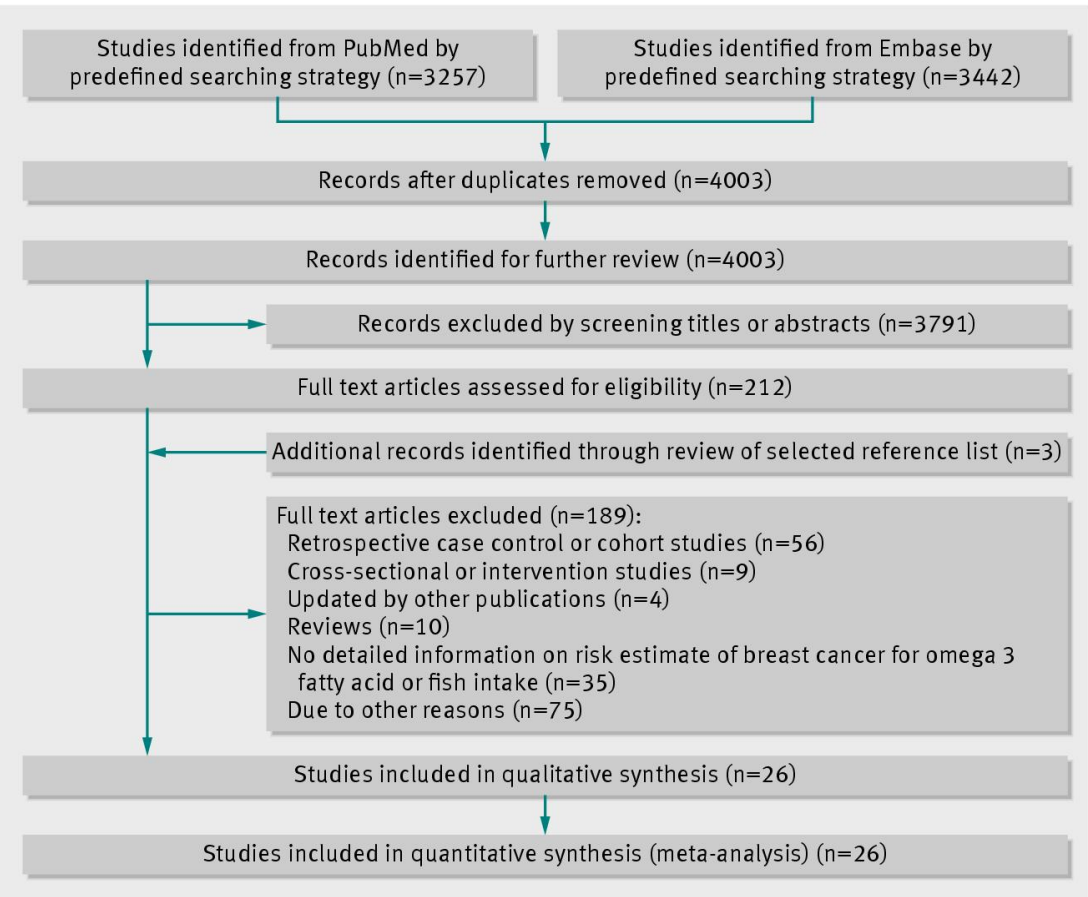

Fig 1 Flow diagram for selection of studies in meta-analysis of intake of polyunsaturated fatty acids and risk of breast cancer

Study

Mills ${ }^{32}$

Vatten ${ }^{28}$

Toniolo ${ }^{42}$

$\mathrm{Key}^{29}$

$\mathrm{Cho}^{20}$

Gago-Dominguez premenopausal ${ }^{12}$

Gago-Dominguez postmenopausal ${ }^{12}$

Stripp 33

Holmes premenopausal ${ }^{30}$

Holmes postmenopausal ${ }^{30}$

Folsom ${ }^{21}$

Wakai ${ }^{5}$

Engeset premenopausal ${ }^{31}$

Engeset postmenopausal ${ }^{31}$

Overall: $P=0.009, I^{2}=54 \%$

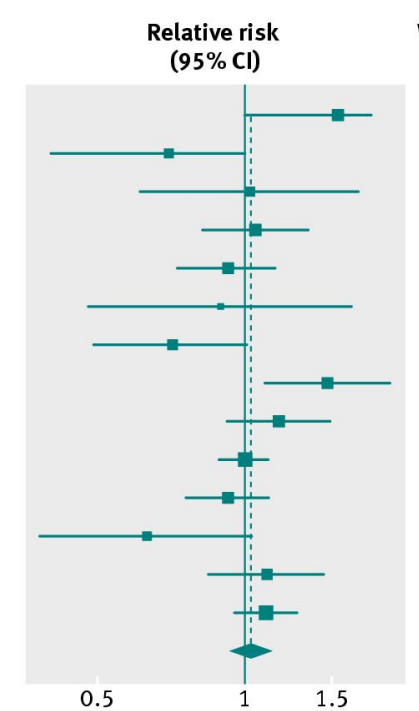

0.5

$\begin{array}{cc}\begin{array}{c}\text { Weight } \\ \text { (\%) }\end{array} & \begin{array}{c}\text { Relative risk } \\ \text { ( } 95 \% \mathrm{Cl})\end{array} \\ 7 & 1.54(1.00 \text { to } 1.81) \\ 4 & 0.70(0.40 \text { to } 1.00) \\ 3 & 1.02(0.61 \text { to } 1.71) \\ 8 & 1.05(0.82 \text { to } 1.35) \\ 9 & 0.92(0.73 \text { to } 1.15) \\ 2 & 0.89(0.48 \text { to } 1.66) \\ 5 & 0.71(0.49 \text { to } 1.01) \\ 7 & 1.47(1.10 \text { to } 1.98) \\ 8 & 1.17(0.92 \text { to } 1.50) \\ 13 & 1.00(0.89 \text { to } 1.12) \\ 10 & 0.92(0.76 \text { to } 1.12) \\ 3 & 0.63(0.38 \text { to } 1.03) \\ 8 & 1.11 \text { (0.84 to } 1.45) \\ 12 & 1.10 \text { (0.95 to } 1.28) \\ 100 & 1.03 \text { (0.93 to } 1.14)\end{array}$

Fig 2 Relative risk of breast cancer for highest $v$ lowest category of dietary fish intake. Overall relative risk calculated with random effects model 


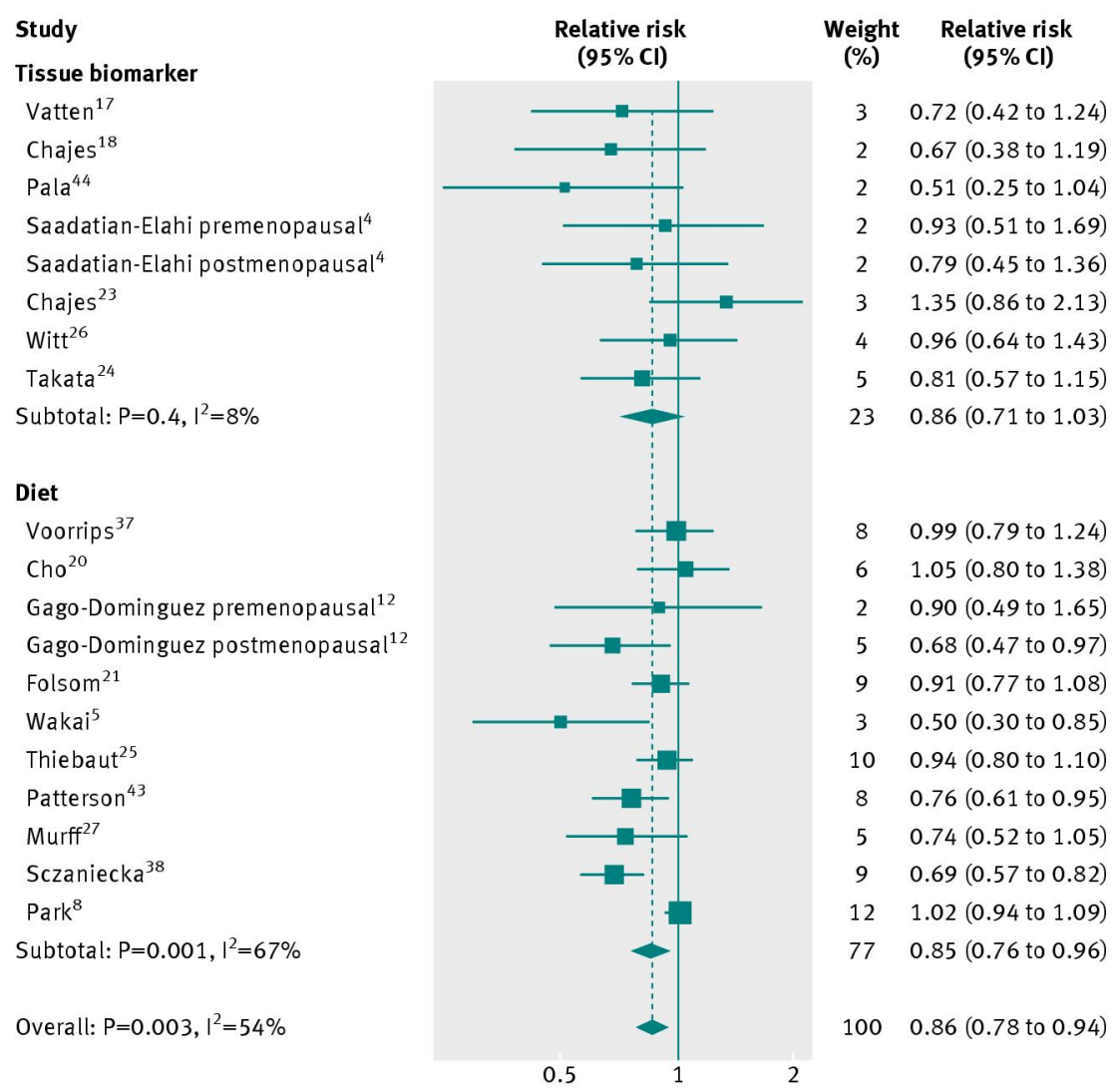

Fig 3 Relative risk of breast cancer for highest $v$ lowest category of marine $n-3$ PUFA. Overall relative risk calculated with random effects model

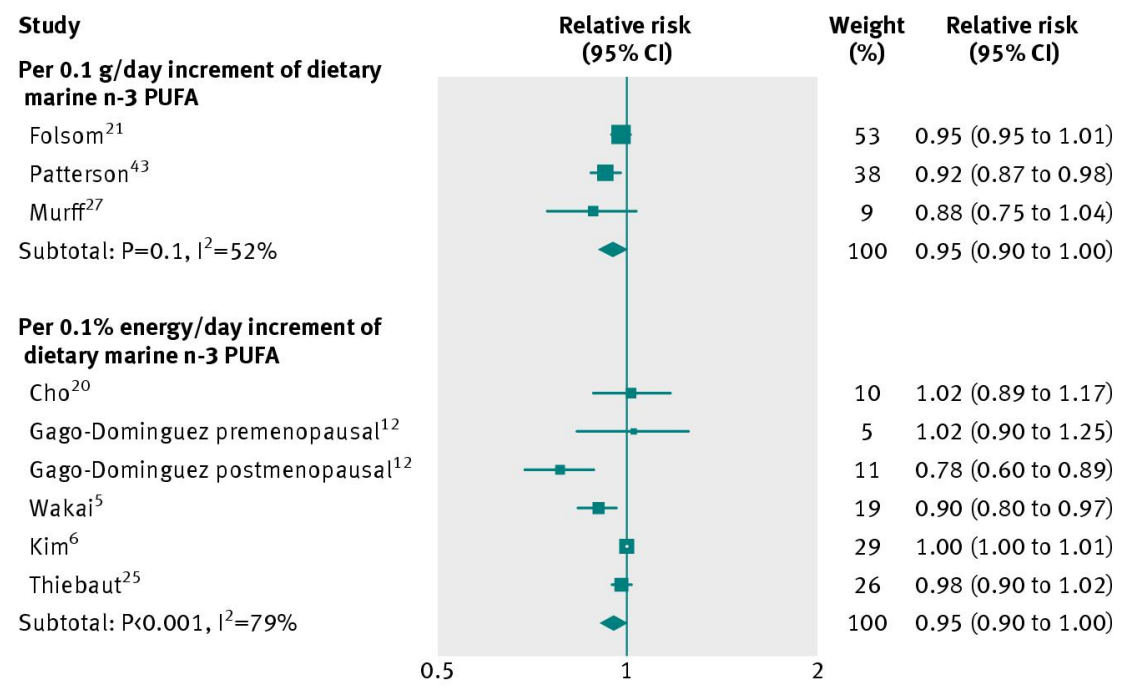

Fig 4 Dose-response meta-analysis for per $0.1 \mathrm{~g} /$ day or $0.1 \%$ energy/day increment in intake of marine n-3 PUFA intake and risk of breast cancer. Overall relative risk calculated with random effects model 


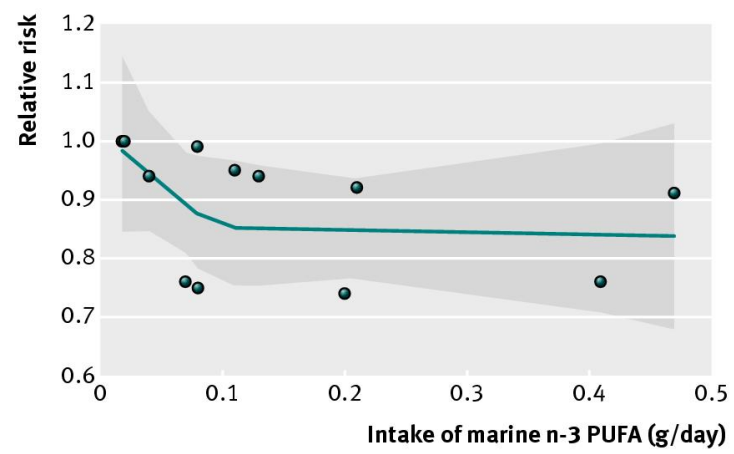

Fig 5 Dose-response analysis for curvilinear association between marine n-3 PUFA intake (g/day) and risk of breast cancer. Shaded area represents $95 \%$ confidence limits for fitted curve. $\mathrm{P}=0.21$ for non-linearity, which indicates no curvilinear association

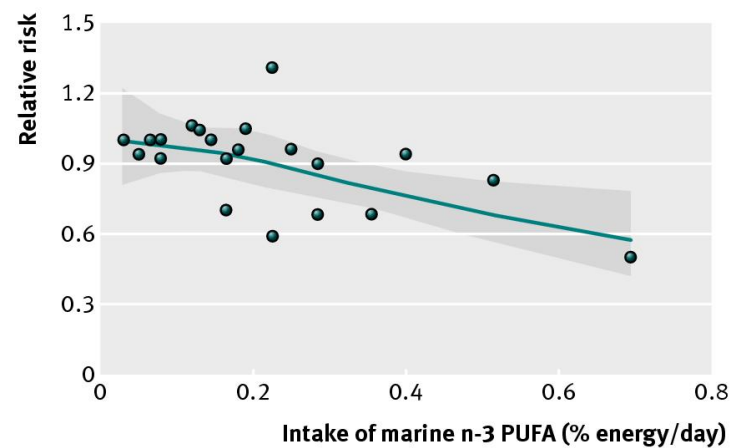

Fig 6 Dose-response analysis for curvilinear association between marine n-3 PUFA intake (\% energy/day) and risk of breast cancer. Shaded area represents $95 \%$ confidence limits for fitted curve. $\mathrm{P}=0.011$ for non-linearity, which indicates no curvilinear association 